\title{
A computer running several psychological laboratories
}

FRANK RESTLE and THOMAS V. BROWN, INDIANA UNIVERSITY, Bloomington, Indiana 47401

Seven psychological laboratories, capable of testing 22 human and 4 animal Ss in seven different experiments using a variety of peripheral equipment, are in various stages of operation using an IBM 1800 process-control computer in the Psychology Department at Indiana University. Each experiment utilizes four appropriate software programs: SETUP, PROCEDURE, UTILITY, and OUTPUT. The programming is usually simple enough to be done by nonprofessional programmers. This simplification was made possible by (1) a set of subroutines written to handle many of the process-control programming complexities, and (2) the IBM-provided Time-Sharing Executive (TSX) program.

In August, 1966, the senior author, along with James G. Greeno and N. John Castellan, received notice that the Public Health Service had approved research grants in which the major equipment was a process-control computer. From March until May, 1967, various components were delivered, and by August, 1967, the first experimental room was operating. By February, 1968 , four rooms were operational, with five rooms now operating under the time-sharing system.

This paper is a review of the purposes, design, equipment, and methods of this system.

\section{PURPOSE AND BACKGROUND}

Our research grants called for studies of memory, concept-attainment, perception, and such mental skills as arithmetic. Because three faculty members and about 20 graduate students were involved, it was appropriate for the computer to serve several functions and to provide a large work capacity. The one computer could provide the controls for several laboratories, and, with time sharing, allow simultaneous experiments in all rooms.

A typical experiment is composed of 100 to 500 discrete trials, possibly broken into blocks separated by short breaks. On each trial, the Ss are shown a stimulus; then they respond and may be given feedback. The form of the stimuli, responses, and feedbacks depends on the experiment. To provide a latitude of choice, each experimental room utilizes different types of apparatus. Consequently, the E may select the room most compatible with his experimental needs.

Programming necessary for a given experiment has been minimized by two features: (1) The time-sharing system provided by IBM served as a basis on which to build a number of subroutines. ${ }^{1}(2)$ The subroutines simplify the programming and provide a systematic framework within which to plan and write programs to control the experiments.

\section{THE CONTROL SYSTEM}

The laboratory is centered around an IBM 1800 real-time process-control computer, designed to record data from a variety of sources, and to provide outputs controlling different types of devices. In addition, it has the usual computer input/output units.

The entire set of input/output units consists of (1) a 1442 card read/punch, (2) an $1816 \mathrm{printer} /$ keyboard, (3) a 1053 printer, (4) a 2848 control unit that drives the six 2260 display stations in the CR room, (5) an 1810 disk unit, (6) a Roytron Model 518 paper-tape punch, (7) the set of Electronic Contact Operates (the solid-state switches that the computer opens and closes as instructed in the programs), and (8) the set of Program Interrupt Contacts (the switches whose closure can be sensed by the computer). The 1810 disk unit provides high-speed bulk storage for programs and data, to be read from the disk into the core storage as needed. The storage capacity of the 1810 is 512,000 16-bit words.

The computer has a core memory of 24,000 16-bit words, with a 2-microsecond cycle time, and has 12 levels of priority interrupts. Software programs include FORTRAN, a Macro Assembler, and a large assortment of subroutines for all the time-sharing functions. Also included are the myriad of subroutines necessary to handle the interrupt conditions and assorted bookkeeping tasks, i.e., storing half-finished programs on the disk, and then executing a program that services a higher-priority interrupt. When the higher-priority program is finished, the half-finished program to be completed is then read back into core memory from the disk. All the above programs were provided by IBM, along with the Time-Sharing Executive System (TSX) that coordinates and monitors all the programs, in addition to handling most input/output and interrupt functions. With TSX, it becomes possible to share the computer's time among its various functions, servicing interrupts when necessary while using the extra time available from the process control to execute such nonprocess programs as compilation or data analysis. Time sharing is necessary for the simultaneous running of more than one laboratory room.

Execution of any program running an experiment in one of the rooms consists mainly of opening and closing the appropriate switches at the proper times, sensing when other switches are closed, handling the storage of responses, and in some experiments, determining which stimulus is to be presented on the basis of past responses by the $\mathrm{S}$. In programming for an experiment, the experimental procedure is broken into a series of intervals. These intervals are of two types, depending on how the interval is terminated. The time interval may be of fixed length, and terminated by a programmed timer that is part of the computer. The second type is the response interval that lasts until a response is made by a S. This response interval would be used, for example, when the $S$ had a forced-choice response. The programmer must determine which functions are to be performed at the beginning and end of each of these intervals (see Fig. 1).

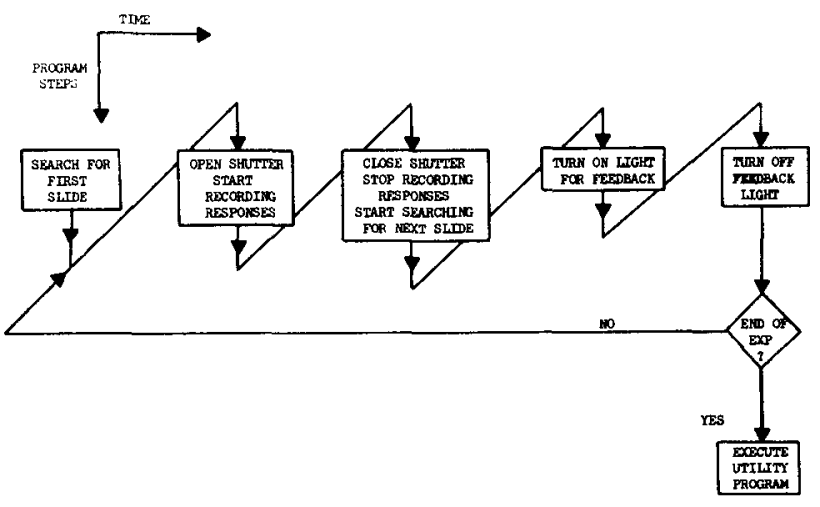

Fig. 1. Relation between the timer intervals and the functions performed. 


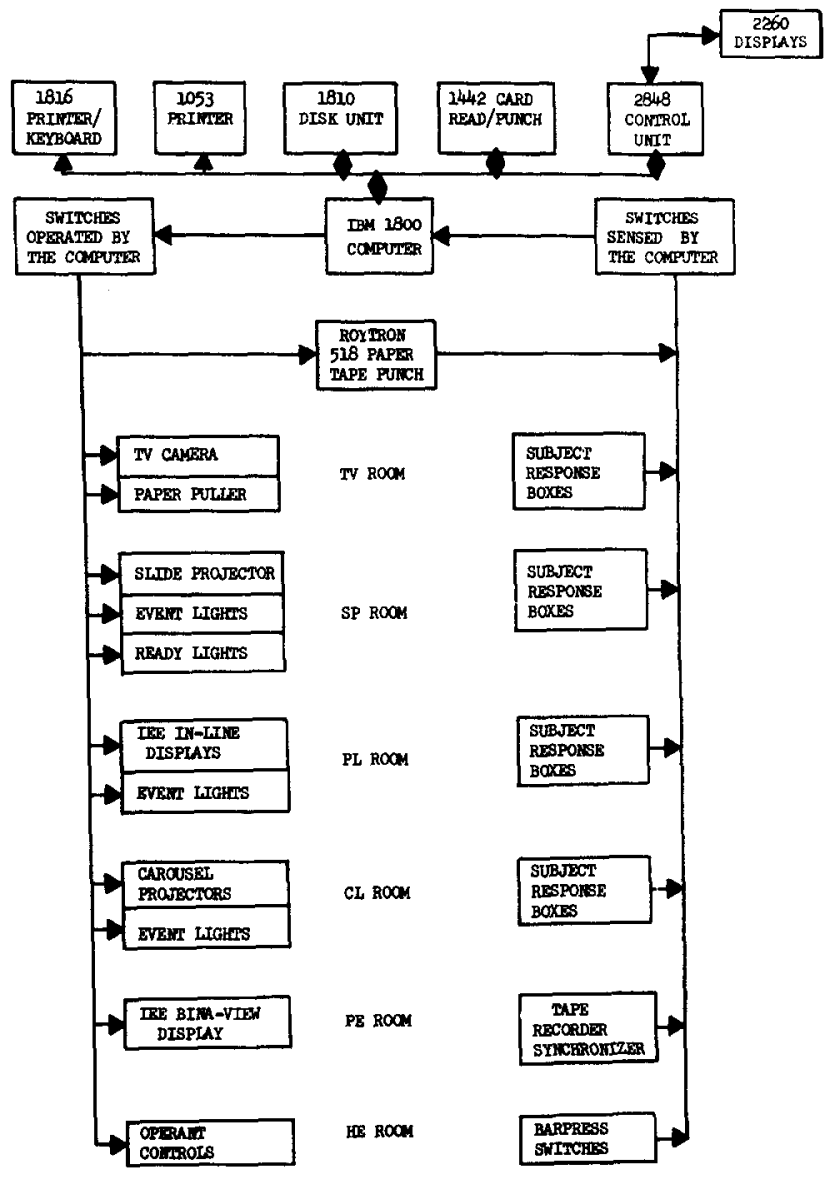

Fig. 2. Overall plan of the computer operation of the various laboratories.

For instance, a stimulus would be turned on at the beginning of a fixed interval, and turned off at the end. Once the functions in the program are executed at the beginning of an interval, the computer ignores that experiment, handling other programs, until the interval is terminated-either by the programmed timer or by a S's response. When no experiment is being serviced, the computer drops back to handling the programs of lower priority.

\section{PERIPHERAL EQUIPMENT}

The computer operates peripheral equipment to send information to the Ss and to sense responses that the Ss make. Any one peripheral system has advantages and limitations, so several different and complementary systems have been installed. Each system is installed in a separate room or suite, and handles four or more Ss concurrently (see Fig. 2).

\section{Slide Projector Room}

The Slide Projector (SP) room uses a Spindler-Saupe model random-access slide projector under computer control mounted outside the $\mathrm{Ss}^{\prime}$ room, providing random access within $5 \mathrm{sec}$ to any of the 48 slides. This provides a gor ntical system for delivering complex displays, including color, a permits the same physical slides to be used, in different random orders, for many experimental sessions. The limitations are that all four Ss see the same display, hence displays cannot be made contingent upon the individual's responses unless only one $S$ is tested at a time. Many studies require that new stimuli be delivered quickly, and the 5 -sec search time is then a source of difficulty. The projector has been used mainly in complex concept-identification studies, and to present figures for perceptual studies.
The SP room provides each $\mathrm{S}$ with a panel that has six response buttons and a small feedback light over each button. These lights are individually computer controlled, so that complex feedback, contingent upon the S's response can be provided. This sytem has been used, without slides, for studies of serial pattern learning.

Finally, since the slide projector is controlled through a panel of relays operated by the computer, it is a simple matter to connect other output devices to those relays. In several recent studies, a bank of tone generators has been constructed so that sequences of tones, either for psychophysical purposes or as patterns, can be presented.

\section{Television Room}

The Television (TV) room uses a two-room suite. In the control room is a paper-pulling system that handles continuous-form computer printout sheets. Stimuli are prepared on such paper, then a closed-circuit TV camera transmits each stimulus to monitors in the Ss' booths in the next room. The four Ss respond with six response buttons on a panel, and feedback, if any, comes from the TV screen. The stimulus lists are generated on the large $\mathrm{CDC} 3600$ computer at the Indiana University Research Computing Center. Lists of great complexity may be generated and used easily, with a new permutation of stimuli (and of conditions) with each session. It is possible to check the stimulus lists before presenting them, thereby avoiding possible difficulties in list-generating programs. Since stimuli are listed on the continuous form, they can be of unlimited complexity and length, yet take up no capacity of the computer. This system is extremely economical of computer effort. Also, the paper puller operates rapidly and can change stimuli in approximately $.10 \mathrm{sec}$, much faster than the slide projector. The system is limited to the line printer's character set, and no contingencies on Ss' responses are possible. This flexible system has been used for studies of verbal learning, short-term memory, arithmetic, memory and visual search, choice reaction time, and choices between gambles.

\section{Probability-Learning Room}

The Probability-Learning (PL) room uses digital in-line displays, one in each booth, as a stimulus and employs a two-response system. These projectors are independent-one for each S-with a choice of any of 12 stimuli, providing almost instantaneous onset of the stimulus. Their limitations are that the number of alternatives is small and the optical quality leaves much to be desired, but the main advantage is that the next stimulus may be contingent on previous responses. Signal lights over the response buttons are also available for feedback, independent of the projectors. This system has been used mainly for studies of probabilistic concept identification.

\section{Cathode-Ray Tube Room}

The Cathode-Ray Tube (CRT) room uses six IBM 2260 display stations, each with an attached typewriter keyboard. Five displays are used by Ss while the sixth is available to the $E$ to monitor the $\mathrm{Ss}^{\prime}$ responses and possibly enter experimental parameters. Ss receive alphanumeric displays, up to six lines of 40 characters each, on the display screen, and type their responses, which are displayed on the screen as typed. This system permits very complex verbal or numerical displays, which are generated independently for each $S$ and therefore can be made dependent on responses. The entire stimulus can be presented within $1 / 30 \mathrm{sec}$, so that very rapid displays are possible (though not at what would be considered tachistoscopic speeds). The complexity of responses permits use of such verbal-learning techniques as noun or nonsense-syllable responses, and studies of free recall have been performed. The system is also suitable for studies of such more complex stimulus material as mathematical problems, puzzles, etc. Its main limitation is the relatively high cost of the 
system, since the 2260 display stations require a 2848 Display Control. Timing arrangements within the system preclude accurate reaction-time measurements. Also, since the stimuli are stored and generated within the computer, a relatively large storage area must be assigned to the CRT room. Communication software for the 1800-2260 linkage was not available and had to be developed by our Systems Analyst.

\section{Concept-Learning Room}

The newest room is the Concept-Learning (CL) laboratory, in which each of four Ss has two rear-projecting Kodak Carousel slide projectors, each equipped with a shutter. The computer can step the projector for each $S$ to the next slide only, having no random access capability, but it does have separate control of the shutters, and of feedback lights over the response buttons. Also, since there are two projectors for each $\mathrm{S}$, the computer can change from one projector to the other, and at that time may shift to stimuli having different dimensions. This laboratory permits the richness and complexity of color slides, and yet permits the different $S s$ to have different stimuli, and to change stimuli individually as they reach a performance criterion. The disadvantage is that the slides cannot be accessed randomly.

\section{Additional Peripheral Systems}

The above are the major permanent installations intended for studies in human learning and performance. It may be remarked that the rooms are all in the Psychology Building, in a modern research wing, and that the computer, SP laboratory, and CRT laboratories, along with an office for the System Programmer, are on the third floor and the TV, PL, and CL laboratories are on the second floor in suites just below the computer.

An additional installation, controlled by the computer, is a set of four Skinner boxes used for rat behavior studies in Professor George Heise's Psychopharmacology Laboratory. At this time, this laboratory is studying Sidman avoidance schedules as a function of various drugs and dosages, with particular interest in interresponse time. An automated training technique for double-lever continuous avoidance is being developed currently, as well as several other complex schedules. The peripheral equipment is standard operant instrumentation, controlled and recorded completely by the computer.

Professor Lloyd Peterson uses an IEE Bina-View display capable of displaying any single letter or number for studies in short-term memory and shadowing. Although the computer controls the stimuli, it does not record responses. Eventually, the Bina-View will be synchronized to a tape recorder in order to locate precisely visual and oral stimuli in time.

The most time-demanding application of the computer was for Professor James Allison's study of the distribution of the times between licks for rats drinking water. This required that events as close as $1 / 10 \mathrm{sec}$ apart be recorded to an accuracy of about $1 \mathrm{msec}$. This has been the only experiment thus far that has required a temporary monopoly of the computer, precluding time sharing.

\section{DOING AN EXPERIMENT}

Every experiment is programmed anew in order to minimize its load on the system. Writing a series of generalized programs, in which the $E$ only selected certain options, would have been too restrictive and inefficient. Although a large programming staff would be helpful in handling the variety of experiments run in the lab, no such staff is available. Each $E$ has the responsibility for writing his own programs, with help from the Systems Analyst. Graduate students and faculty who are acquainted with FORTRAN can usually write their own experimental programs with a minimum of aid and instruction because nearly all of the powerful features of the system are incorporated via subroutines that are written so as to require a minimum knowledge of the system from the programmer. The present system divides the programming task into logically separate parts that may be written relatively independently.

Each experiment uses four programs, called here: SETUP, PROCEDURE, UTILITY, and OUTPUT. Of these, SETUP and OUTPUT are nonprocess programs. That is, they do not involve any connections to the experimental room. SETUP is run before the experiment starts, setting up files on the disk, storing stimuli on the disk, and doing whatever initialization is necessary for the experiment. OUTPUT is run whenever the response files on the disk are filled with data that is either punched on cards, listed on the printer, or analyzed.

PROCEDURE directly controls the experiment while it is running, while UTILITY performs a variety of "housekeeping" functions necessary from time to time while the experiment is in operation. PROCEDURE resides in core memory all the time in a reserved area called the SKELETON. The only time that PROCEDURE is executed is when an interrupt involving the experimental room is made.

Interrupts may happen in two ways: (1) when an interval timer interval to the computer times out, and (2) when a response button is pressed. Each room has its own interval timer that can be set for any time period. When this period times out, an interrupt entry is made into the PROCEDURE program for that room. The programming facility is available to cause an entry into the PROCEDURE program each time a response button in the room is pressed, but for most experiments this is not necessary.

Each PROCEDURE program has a work area of core called INSKEL COMMON, in which limited amounts of stimuli, data, and parameters can be stored. One of the functions of UTILITY is to store the data from this area on the disk, and load it with more stimuli from the disk, as is necessary from time to time, simultaneously with the experiment in progress.

Since PROCEDURE programs are time sensitive and therefore operate at high priority in the time sharing, each PROCEDURE program is kept as short as possible. Its size is kept small because it resides in core memory all the time (in contrast to UTILITY, which is read into core and executed only when needed). Generally, one pass through PROCEDURE, to process an interrupt, requires only 20 to $50 \mathrm{msec}$.

Since each trial involves several discrete intervals to be timed, PROCEDURE has to keep track of which interval is being timed. This is done with a FORTRAN variable, called IWHER, which is set equal to a number denoting the interval of the trial that is being timed. For example, if a slide is to be selected for the projector in the SP room, $5 \mathrm{sec}$ must be allowed for searching before the shutter is opened to present the slide. IWHER would be set equal to 1 , and the timer would be set for $5 \mathrm{sec}$. At the end of $5 \mathrm{sec}$, PROCEDURE would be entered and the value of IWHER would determine which branch of the program would be executed. In this case, IWHER $=1$ would cause a branch to the section of the program that opened the shutter. If the shutter is to remain open for $3 \mathrm{sec}$, IWHER would be set equal to 2 , and the timer set for $3 \mathrm{sec}$. At the end of the $3 \mathrm{sec}$, IWHER $=2$ would cause a branch to the section that closed the shutter. The last interval of the trial would be timed with IWHER set to 1 so that the trial times would recyde.

At the end of each time interval, a number of functions must be performed, such as illustrated above, as well as checking the trial number to determine whether INSKEL COMMON has been filled so that a call to UTILITY would be necessary, testing for the end of the experiment, and various housekeeping tasks. After the interval timer is set and running, control is turned over to the time-sharing system to make the computer available to handle 
other programs until one of the interval timers times out.

UTILITY handles the relatively time-consuming aspects of running the experiment, such as reading and writing from the disk. Each experimental room has a monitor box, with "start," "wait," and "abort" buttons that the $E$ uses to control the experiment. When the E presses his "start" button in his experimental room, this causes an interrupt that calls UTILITY into core from the disk and executes it. UTILITY recognizes that this is its first call so that it performs initialization of the experiment. When this is accomplished, UTILITY turns control over to PROCEDURE, and the sequence of trials is begun. At various times during the experiment, PROCEDURE calls UTILITY back into core to dump data on the disk, and read more stimuli from the disk into INSKEL COMMON. When this is done, control is returned to PROCEDURE for a continuation of the experiment. There are ways to avoid the delay in the experimental procedure (rarely over 2 or $3 \mathrm{sec}$ ) by more complex programming, but this has not been necessary. At the end of the experiment, UTILITY is called in to do the housekeeping necessary to terminate the experiment.

Most of the complexity of the time-sharing programming is handled by the Master Interrupt Servicing program PIINT, which was written by our Systems Analyst. This program was written to provide a link between TSX, the IBM-provided time-sharing system, and the process programs written by Es. Among its numerous functions, it handles the interrupts caused by a S's pressing a response button, keeping a record of this response in INSKEL COMMON to be picked up and stored by PROCEDURE. It also measures response times, if the $\mathrm{E}$ needs this measure. The program also handles the initialization of the experimental session by determining which "start" button was pressed, and executing the UTILITY program for that room. In addition to the "start" button, the $\mathrm{E}$ may abort, or temporarily pause the session by pressing the "abort" or "wait" button. PIINT handles these requests, taking the burden from the programmer.

Among the other functions performed by PIINT is that it turns on indicator lights above the computer console, indicating which experimental rooms are in operation.

Transfer between the various programs is made possible by the TSX system, with TSX doing the necssary storage allocation and bookkeeping. The TSX system also allows the efficiency of the priority-interrupt system to be utilized with a minimum of programming. For example, the PROCEDURE routines are the most sensitive to time delays. If one room's UTILITY program took $10 \mathrm{sec}$ to execute, and it began immediately after a 1 -sec stimulus was turned on, then without the interrupt system, it would be at least $10 \mathrm{sec}$ before the stimulus was turned off. With the interrupt system, the PROCEDURE programs have the highest priority, with the UTILITY and other more time-consuming programs being assigned a lower priority level. Thus, if a timer runs out while a long UTILITY program is being executed, the execution of the lower-priority program is temporarily halted. A higher-priority program is then executed, followed by a return to the place where it left off in the lower-priority program. The priority-interrupt design of the computer converts it into a process-control computer, able to handle high-priority situations immediately, regardless of how long it takes for the current program to finish. Without interrupts, long programs would have to run to completion before any other function could be performed by the computer.

\section{PROCEDURE SUBROUTINES}

The PROCEDURE program is generally the most difficult of the four programs to write. To ease this, the various stimuli are turned on/off by use of subroutines called from the FORTRAN-written PROCEDURE. For example, in order to turn on Event Light 3 in Booth 4 in the SP room (Room 2), the programmer writes CALL EVN2 $(4,3)$, which calls subroutine EVN2. This subroutine determines the switch control light, 3 , for Booth 4 in the SP room, and operates that switch. To select a slide, No.36, in the slide projector in the SP room, the programmer writes CALL SERCH(36). The subroutine converts the 36 to a binary pattern appropriate for the hardware of the slide projector, and operates the combination of switches necessary to select this slide. Two-way interaction with peripheral hardware is illustrated by subroutine PAPUL, which spaces the paper that contains the stimuli for the TV room. The instruction CALL PAPUL(2) causes the motor that moves the paper to start in its spacing of two lines. Instead of tying up the computer for the $1 / 10 \mathrm{sec}$ necessary to move the paper, only the command is given to start the motor and the program continues. A cam, attached to the motor's shaft, operates a microswitch that causes an interrupt whenever one line is spaced, so that after two lines are spaced, the command is given to turn off the motor. PINT handles this feedback from the motor, turning it off after the correct number of interrupts are counted.

All subroutines are documented in our laboratory manual for reference when writing programs, so that new programmers are easily introduced to the various features of the system.

Figure 3 gives an abbreviated example of a FORTRAN-written procedure program.

\section{CURRENT PERFORMANCE}

The present laboratory operation consists of five separate laboratory rooms, containing four, four, four, and five booths, and four Skinner rat-boxes. A large share of the laboratory's operation has been with at least three rooms operating simultaneously. Even when all five rooms were operating simultaneously, no perceivable time delays in the procedures were observed, even when there was time sharing with background (nonprocess) computing. Although some problems arose in the beginning with the rooms' interacting with each other, programming methods and procedures have eliminated these problems. No irregularities in experimental procedures due to running several experiments at the same time have been found.

A measure of the load on the system is the amount of its time available for such nonprocess programs as compilation, listing, data analysis, etc. Noticeable delays occur in the nonprocess programs only three or four times an hour when all the rooms are in operation.

Certain types of errors in the programs executed will cause a halt, reset, and reloading of the system, with the consequence that all experiments running at that time are lost or "bombed." When this occurs, all Ss are dismissed, and the next group is run as scheduled. The Systems Analyst developed a method whereby experiments can be debugged in the nonprocess mode prior to placing them on-line and this "bombing" problem has been virtually eliminated. Following the rule that no programs are executed for the first time while the experiments are running has helped in elimination of this problem since nearly all the serious errors in a program show up the first time it is executed. The new multiprogramming system for the 1800 will replace the TSX system in a few months, so that even with the serious errors, no experiments will be "bombed."

Along with operating discipline, which must be high, there is the problem of developing and executing experiments. Thus far, it appears that any student capable of gaining reasonable mastery over FORTRAN is able to write his own experimental programs. Generally speaking, when radical departures from previous procedure are required, experienced graduate students and the Systems Analyst join in the work. Most new experiments actually turn out to be minor deviations from some previous experiment, 
FORTRAN

Instruction

Comments

GO TO $(1,2,3,4)$, IWHER

1 CALL OPEN (2)

CALL LETGO $(2,7)$

IWHER $=2$

GO TO 100

2 CALL CLOSE (2)

CALL NOGO $(2,7)$

from Room 2.

CALL SEARCH (ISLID(N)) Starts slide projector searching for next slide-number ISLID(N) where $\mathrm{N}$ is trial number.

JRESP(N) $=$ IRESP

IWHER $=3$

GO TO 100

3 CALL EVN2 (7, NANS(N))

Turns on correct response light for Trial $\mathrm{N}$ for all Ss.

IWHER $=4$

Set up return for end of timed interval.

\section{GO TO 100}

4 CALL EVF2 (7, NANS(N))

IF (NMAX - N) 5,5,6

5 CALL LEVEL (8)

\section{RETURN}

$6 \mathrm{~N}=\mathrm{N}+1$

IWHER $=1$

100 CALL COUNT

(2,2,ITIME(IWHER))

Which interval was just Opens shutter in Room 2 (SP room).

Permits responses to be recorded in all booths in Room 2.

Set up return for end of timed interval.

Closes shutter in Room 2 (SP room).

Responses are transferred from where they are stored when they come in into storage for that trial.

Set up return for end of timed interval.

Turns off correct response light.

Test for end of experiment.

Calls utility program which is on Level 8.

Exit from this program.

Increment trial number.

Sets timer to value ITIME(IWHER) and starts it, to return to procedure program for Room 2.

Exit from this program.

Fig. 3. Abbreviated procedure routine.

example of a

and the programs are more copied than invented. When a particular experiment becomes established as an important basic procedure, then attention is turned to refining its procedure and making it more efficient. In general terms, it can be said that the idea of having Es do their own programming has been successful in this laboratory.

The most complex procedures have taken a week or two to become operational. Many experiments, when their procedures are conventional, can be placed in operation within 2 or 3 days after flow charting. Since programming is naturally a cooperative enterprise, it is not possible to say just what a naive or an experienced programmer can accomplish, but the expected delays in programming experiments have not appeared. This seems to be because the programmer and $\mathrm{E}$ are in good communication, being the same person, and the result is that many experiments are extremely simple and direct, designed to do a specific task, rather than being conceived as monumental general systems.

\section{PERSONNEL}

The work in the Mathematical Models Laboratory revolves around the research of the three faculty members who are associated with the laboratory. Primary responsibility for programming, as well as for maintaining the system, belongs to the Systems Analyst. The dozen or so graduate students, assistants, and fellows run the faculty member's experiments and their own, working with the Systems Analyst in the programming tasks. Hardware construction, modification, and maintenance is done by the graduate assistants working in the lab, with some of the more difficult problems handled by the departmental shop men.

\section{costs}

The present system is partly rented and partly on a 3-year time-purchase plan. Payments to IBM amount to nearly $\$ 3,400.00$ per month, including maintenance. In addition, there is apparatus controlled by the computer with a purchase value of approximately $\$ 4,000.00$. Site preparation cost approximately $\$ 4,000.00$, including the air conditioning. Operating costs consist almost entirely of salaries.

\section{CONCLUSIONS}

Most psychologists will have to justify the expense of a medium-sized computer in their laboratories. The justifications made for this system are (1) a great increase in experimental accomplishment, both in quantity and quality, and particularly an increase in graduate-student research, (2) an improvement in experimental technique, particularly in more direct measurements of the variables of interest, and improved experimental design (randomizing irrelevant variables, more complete analysis of data, etc.), and (3) the ability to pursue experimental questions far beyond the usual single experiment.

The last of these three points is of great importance. It is common to decide, after having finished an experiment, that it really should have been done differently, or to discover in the data a "post-hoc" indication more interesting than the original question. With the computerized laboratory, it is usually a simple matter to revise the procedure and do the next, more interesting, study. If, as in this laboratory, an experiment requires only about a week of half-time experimenting, and if preparing materials, running the experiment, and analyzing the data are easy, then the $\mathrm{E}$ is motivated to continue pursuing his problem and refining his technique until he has a clear and definite answer. Such results are, of course, easy to report as compared with the usual 
experiment, and it is not necessary to squeeze a publication out of each preliminary study.

An additional justification is the ability to perform experiments using an active interaction between apparatus and $S$. The eventual possibilities for such studies are tremendous, but some difficulty has been experienced in that theoretical resources seem, at present, inadequate. Interactive experiments are easily designed and performed, but it is not as easy to interpret the results, since thery has been little experience with the new methodological questions introduced by the interaction.

\section{FUTURE DEVELOPMENT}

When planning began, a detailed time schedule was established, from installation of the equipment up to the time when all laboratories would be operating on a time-shared basis. Despite the many uncertainties, the predictions were reasonably accurate, though conservative. In terms of requirements, however, original plans were found to be seriously conservative, and it was necessary to increase core memory from the original 8,000 words to 16,000 words in order to begin using TSX. Later changes were to increase core to 24,000 words, and to increase the number of switch inputs and outputs as well as to change from a lower (4-microsecond cycle time) to faster (2-miscrosecond) machine speed. The present system operates adequately.

Extension of the grant, now with Restle and Castellan and the cooperation of Richard Shiffrin and George Heise, is the occasion for some changes in operating system and equipment. The main change will be to shift from TSX time-sharing executive to MPX multiprogramming system. This will permit a considerable increase in flexibility in programming. TSX was found to be suitable for a fixed application in which a complex multichannel operation is to be established and then used for a long time. In a research laboratory, there is reason to change one or another experiment almost daily. A change of an experiment requires what is called a "SKELETON BUILD" operation in which the skeleton core area is assigned to its various tasks, the remaining area of core is apportioned as variable core, and numerous other functions are performed. This requires nearly $30 \mathrm{~min}$ to perform if no error creeps in, and requires that no processes be running during this time. The new MPX system makes dynamic allocations of skeleton and variable core, and makes it possible to do a "SKELETON BUILD" while the experiments are in operation. This MPX system, as can be seen, fits the general plan of the laboratory.

Introducing MPX requires use of two disk-storage drives, instead of the one now in operation. At the same time, there will be a change to a faster disk system, because that will permit disk-transfer operations to be used more often during experiments.

Operation for the first 3 years was without a line printer, and the result has been that (1) programming is inhibited because of the inability to list long programs efficiently, and (2) printed indicators could be used for debugging only with extra problems. In addition, it is hoped that simple data-output programs, to show the $\mathrm{E}$ how his experiment is progressing, will be much more convenient using the line printer. Finally, plans have been made to use the new line printer to produce experimental materials for the TV system, thus enabling the use of special printing techniques (such as printing on the back of paper to avoid lines, and ensuring fresh carbon sheets when doing TV printing) and also the ability to store, on the computer disk, the stimulus list to be shown in the TV room. This can be a great aid in scoring and analyzing the data from that apparatus.

Up to the present, it does not seem that the limits of the computer, as such, have been pushed, and the work load will probably be increased. Expansion to control studies in perception and social interaction, to handle observations in animal behavior experiments, and many other such tasks are feasible with the present configuration. As the technical capacity of the laboratory increases, and other faculty members and students become familiar with its possibilities, expansion into new projects seems assured. However, even without such expansion, is appears that the computer, despite its cost, can now be justified as an economical method of controlling laboratory procedures.

The economics of the situation are not justly understood if one compares the computer with "no computer." If the work is to be done without a computer, then it presumably must be done some other way. The peripheral equipment would cost the same whether connected to a computer or not. Much of the control must be automatic if the present experiments are to be done, and this requires a "wired program" computer or logic network, with timers, cables, etc. Such systems have been used in this laboratory, and they have been found expensive (in time, skilled labor, and lost experimental opportunities) to program and reprogram, thereby producing some rigidity of procedure. What is more, the equipment itself is relatively expensive; and five or six such systems would cost a significant fraction of the cost of the computer that can perform the function of numerous separate control systems. Furthermore, one key function of any automatic apparatus is to store the response data. Most separate control systems use punched paper tape, punched cards, or a printout counter to store the data if it is stored in digital form at all. Other systems use event recorders or cumulative recorders, which provide a graphical, but not a numerical, record of the data. Such systems soon inundate Es under masses of undigested data. The computer is uniquely capable of storing, arranging, and analyzing data as they come in, hence permitting the $E$ to progress as rapidly as he can run experiments, rather than being held to the usually slower speed of data anlysis. When production differences are estimated accurately, and costs appropriately calculated, it seems that the multiprogrammed laboratory control computer is reasonably economical for purposes like those of our laboratory.

NOTE

1. Written by our Systems Analyst, Mr. Jerry L. Gordon. 